\title{
Application of Contractive-like Mapping Principles to Fuzzy Functional Differential Equation
}

\author{
$\mathrm{Vu} \mathrm{Ho}^{1, *}$ \\ ${ }^{1}$ Faculty of Mathematical Economics, Banking University of Ho Chi Minh City, Vietnam
}

\begin{abstract}
In this paper, we prove the existence and uniqueness of solution for the fuzzy functional differential equation under generalized Hukuhara derivative via contractive-like mapping principles.
\end{abstract}

Keywords: generalized Hukuhara derivative; contractive mapping; fuzzy differential equation.

2010 Mathematics Subject Classification: 34F05, 34G20, 34A12, 26E50.

\section{Introduction}

Fuzzy differential equations (FDEs) forms a suitable setting for the mathematical modeling of real-world problems in which uncertainty or vagueness pervades. A rich collection of results from the theory of FDEs is contained in the monograph of Lakshmikantham and Mohapatra [1] and references therein.

The method of fuzzy mapping was initially introduced by Chang and Zadeh [2]. Later, Dubois and Prade [3, 4] presented a form of elementary fuzzy calculus based on the extension principle [5]. Puri and Ralescu [6] suggested two definitions for the fuzzy derivative of fuzzy functions. The first method was based on H-difference notation and was further investigated by Kaleva [7]. Several approaches were later proposed for FDEs and the existence of their solutions (e.g. [8, 9]). There are several approaches to the study of fuzzy differential equations. One popular approach is based on H-differentiability. The approach based on $\mathrm{H}-$ derivative has the disadvantage that it leads to solutions which have an increasing length of their support.

Bede and Gal [10] solved the above mentioned approach under strongly generalized differentiability of fuzzy-number-valued functions. In this case the derivative exists and the solution of FDEs may have decreased the length of the support, but the uniqueness is lost. Other researchers have proposed several approaches to the solutions of FDEs (e.g. [11-14],[15]). Almost authors applied the fixed point theorems like the Darbo's theorem and the classical Banach fixed point theorem [16], Schauder's fixed point theorem [17] and the method of successive approximations [8],... as a tool to prove the existence and uniqueness of the solution of FDEs. In [13], Lupulescu proved the local existence and uniqueness via the method of successive approximations and for global existence and uniqueness via the Banach fixed-point theorem. Allahviranloo et al. [17] also investigated existence and uniqueness of the solution of nonlinear fuzzy Volterra integral equations. Using Arzela-Ascoli's theorem and Schauder's fixed point theorem, authors proved thr existence and uniqueness of the solution for this kind

\footnotetext{
*e-mail: vuh@buh.edu.vn
} 
of equations. In [14], using the contractive-like mapping principles, authors discussed the existence and uniqueness of solution of fuzzy differential equation under generalized Hukuhara derivative.

In this paper, we prove the existence and uniqueness of solution for fuzzy functional differential equation under generalized Hukuhara derivative via the some fixed point theorems, established in [18].

This paper is organized as follows: In Section 2 we shall review some results the existence and uniqueness of fixed points for mappings defined in partially ordered sets. Also, we recall some fundamental results of fuzzy sets, ordering relations over fuzzy sets and fuzzy distance, which will be necessary to study the existence and uniqueness of solution for the fuzzy functional differential equation. In Section 3 we study on the existence and uniqueness of solution for the fuzzy functional differential equation.

\section{Preliminaries}

In this section, we shall review some results the existence and uniqueness of fixed points for mappings defined in partially ordered sets. Also, we recall some fundamental results of fuzzy sets, ordering relations over fuzzy sets and fuzzy distance.

Definition 2.1 (see [18]) An altering distance function is a function $\Lambda: \mathbb{R}_{+} \rightarrow \mathbb{R}_{+}$which satisfies

(i) $\Lambda$ is continuous and non-decreasing.

(ii) $\Lambda(t)=0$ if and only if $t=0$.

Theorem 2.1 (see [18]) Let $(X, \leq)$ be a be a partially ordered set and suppose that there exists a metric $d$ in $X$ such that $(X, d)$ is a complete metric space. Let $F: X \rightarrow X$ be a monotone non-decreasing mapping such that

$$
\Lambda(d(F(x), F(y))) \leq \Lambda(d(x, y))-\Upsilon(d(x, y)), \text { for all } x \geq y,
$$

for some altering distance functions $\Lambda$ and $\Upsilon$. Suppose that either $F$ is continuous or $X$ is such that

if a non-decreasing sequence $\left(x_{n}\right)_{n \in \mathbb{N}} \rightarrow 0$ in $X$, then $x_{n} \leq x$ for all $n \in \mathbb{N}$.

If there exists $x_{0} \in X$ with $x_{0} \leq F\left(x_{0}\right)$, then $f$ has a fixed point.

Theorem 2.2 (see [18]) Let $(X, \leq)$ be a be a partially ordered set and suppose that there exists a metric $d$ in $X$ such that $(X, d)$ is a complete metric space. Let $F: X \rightarrow X$ be a monotone non-decreasing mapping such that

$$
\Lambda(d(F(x), F(y))) \leq \Lambda(d(x, y))-\Upsilon(d(x, y)), \text { for all } x \geq y,
$$

for some altering distance functions $\Lambda$ and $\Upsilon$. Suppose that either $f$ is continuous or $X$ is such that

if a non-increasing sequence $\left(x_{n}\right)_{n \in \mathbb{N}} \rightarrow 0$ in $X$, then $x_{n} \geq x$ for all $n \in \mathbb{N}$.

If there exists $x_{0} \in X$ with $x_{0} \geq F\left(x_{0}\right)$, then $F$ has a fixed point. 
Let $\mathscr{K}_{c}\left(\mathbb{R}^{d}\right)$ denote the collection of all nonempty compact and convex subsets of $\mathbb{R}^{d}$. The addition and scalar multiplication in $\mathscr{K}_{c}\left(\mathbb{R}^{d}\right)$, we define as usual, i.e. $A, B \in \mathscr{K}_{c}\left(\mathbb{R}^{d}\right)$ and $\lambda \in \mathbb{R}$, then we have $A+B=\{a+b \mid a \in A, b \in B\}, \lambda A=\{\lambda a \mid a \in A\}$. The Hausdorff metric $d_{H}$ in $\mathscr{K}_{c}\left(\mathbb{R}^{d}\right)$ is defined as follows

$$
d_{H}(A, B)=\max \left\{\sup _{a \in A} \inf _{b \in B}\|a-b\|_{\mathbb{R}^{d}}, \sup _{b \in B} \inf _{a \in A}\|a-b\|_{\mathbb{R}^{d}}\right\},
$$

where $A, B \in \mathscr{K}_{c}\left(\mathbb{R}^{d}\right)$. It is known that $\left(\mathscr{K}_{c}\left(\mathbb{R}^{d}\right), d_{H}\right)$ is a complete metric space.

Denote $E^{d}=\left\{u: \mathbb{R}^{d} \rightarrow[0,1]\right.$ such that $x(z)$ satisfies (i)-(iv) stated below $\}$

(i) $\quad x$ is normal, i.e, there exists an $z_{0} \in \mathbb{R}^{d}$ such that $x\left(z_{0}\right)=1$;

(ii) $x$ is fuzzy convex, that is, for $0 \leq \lambda \leq 1, x\left(\lambda z_{1}+(1-\lambda) z_{2}\right) \geq \min \left\{x\left(z_{1}\right), x\left(z_{2}\right)\right\}$, for any $z_{1}, z_{2} \in \mathbb{R}^{d}$;

(iii) $x$ is upper semicontinuous;

(iv) $[x]^{0}=\operatorname{cl}\left\{z \in \mathbb{R}^{d}: x(z)>0\right\}$ is compact.

Then $E^{d}$ is called the space of fuzzy sets.

For $\alpha \in(0,1]$, denote $[x]^{\alpha}=\left\{z \in \mathbb{R}^{d} \mid x(z) \geq \alpha\right\}$. We will call this set an $\alpha$ - cut $(\alpha-$ level set) of the fuzzy set $x$. For $x \in E^{d}$ one has that $[x]^{\alpha} \in \mathscr{K}_{c}\left(\mathbb{R}^{d}\right)$ for every $\alpha \in[0,1]$.

The supremum on $E^{d}$ is defined by

$$
d_{\infty}\left(x_{1}, x_{2}\right)=\sup _{\alpha \in[0,1]} d_{H}\left(\left[x_{1}\right]^{\alpha},\left[x_{2}\right]^{\alpha}\right)
$$

for every $x_{1}, x_{2} \in E^{d}$, and $\left(E^{d}, d_{\infty}\right)$ is a complete metric space.

Define $D: E^{d} \times E^{d} \rightarrow \mathbb{R}_{+}$by the expression

$$
D(x, y)=\sup _{t \in[a, b]} d_{\infty}\left(x_{1}(t), x_{2}(t)\right)
$$

It is easy to see that $D$ is a metric in $E^{d}$. In fact, $\left(E^{d}, D\right)$ is a complete metric space.

For every $x, y, z \in E^{d}$ and $\lambda \in \mathbb{R}_{+}$, we have

$$
\begin{aligned}
& D(x+z, y+z)=D(x, y) \\
& D(\lambda x, \lambda y)=\lambda D(x, y) \\
& D(x, y) \leq D(x, z)+D(y, z)
\end{aligned}
$$

Definition 2.2 ([10]) Let $x:(a, b) \rightarrow E^{d}, t \in[a, b]$. We say that $x$ is differentiable at $t$, if there exists $x^{\prime}(t) \in E^{d}$ such that

(i)for all $h>0$ sufficiently small, there exist $x(t+h) \ominus x(t), x(t) \ominus x(t-h)$ and the limits

$$
\lim _{h \rightarrow 0^{+}} \frac{x(t+h) \ominus x(t)}{h}=\lim _{h \rightarrow 0^{+}} \frac{x(t) \ominus x(t-h)}{h}=x^{\prime}(t)
$$

or

(ii)or all $h>0$ sufficiently small, there exist $x(t) \ominus x(t+h), x(t-h) \ominus x(t)$ and the limits

$$
\lim _{h \rightarrow 0^{+}} \frac{x(t) \ominus x(t+h)}{-h}=\lim _{h \rightarrow 0^{+}} \frac{x(t-h) \ominus x(t)}{-h}=x^{\prime}(t) .
$$


In the sequel, we define the following partial orderings $\leq$ and $\leq$ in the space $E^{d}$ and $E^{1}$ :

$$
x, y \in E^{d}, x \leq y \Longleftrightarrow[x]^{\alpha} \subseteq[y]^{\alpha}, \text { for all } \alpha \in[0,1],
$$

and

$$
\begin{array}{ll}
x \leq y \Longrightarrow x+z \leq y+z, & \text { for } x, y, z \in E^{1} \\
x \leq y \Longrightarrow x+z \leq y+z, & \text { for } x, y, z \in E^{d}
\end{array}
$$

and for fuzzy functions,

$$
\begin{aligned}
& f \leq g \Longrightarrow \int_{0}^{t} f(s) d s \leq \int_{0}^{t} g(s) d s, \quad \text { for } t \in[0, a], f, g \in C\left([0, a], E^{1}\right), \\
& f \leq g \Longrightarrow \int_{0}^{t} f(s) d s \leq \int_{0}^{t} g(s) d s, \quad \text { for } t \in[0, a], f, g \in C\left([0, a], E^{d}\right) .
\end{aligned}
$$

\section{Main result}

For a given $\sigma>0$, we denote by $C_{\sigma}$ the space $C\left([-\sigma, 0], E^{d}\right)$. Also, we denote by

$$
D_{\sigma}(x, y)=\sup _{t \in[-\sigma, 0]} d_{\infty}(x(t), y(t))
$$

the metric on the space $C_{\sigma}$. Let $x(\cdot) \in C\left([-\sigma, \infty), E^{d}\right)$. Then, for each $t \in[0, \infty)$ we denote by $x_{t}$ the element of $C_{\sigma}$ defined by $x_{t}(s)=x(t+s), s \in[-\sigma, 0]$.

In this section, we consider the fuzzy functional differential equation under generalized Hukuhara derivative as follows:

$$
\begin{cases}u^{\prime}(t)=f\left(t, u_{t}\right), & t \in[0, a] \\ u(t)=\varphi(t), & t \in[-\sigma, 0] .\end{cases}
$$

Theorem 3.1 (see [12]) A fuzzy mapping $u:[-\sigma, a] \rightarrow E^{d}$ is a solution to the problem (1) on $[-\sigma, a]$ if and only if $u$ is a continuous fuzzy mapping and it satisfies to one of the following fuzzy integral equations:

(i) If $u$ is (i)-differentiable on $[0, a]$, then

$$
u(t)= \begin{cases}\varphi(t), & \text { for } t \in[-\sigma, 0], \\ \varphi(0)+\int_{0}^{t} f\left(s, u_{s}\right) d s, & \text { for } t \in[0, a],\end{cases}
$$

(ii) If $u$ is (ii)-differentiable on $[0, a]$, then

$$
u(t)= \begin{cases}\varphi(t), & \text { for } t \in[-\sigma, 0], \\ \varphi(0) \ominus(-1) \int_{0}^{t} f\left(s, u_{s}\right) d s, & \text { for } t \in[0, a] .\end{cases}
$$

Definition 3.1 (see $[?]$ ) A lower solution for the problem (1) is a function $\mu \in$ $C\left([0, a], E^{d}\right) \cap C^{1}\left([-\sigma, a], E^{d}\right)$ such that

$$
\mu^{\prime}(t) \leq f\left(t, u_{t}\right), \quad t \in[0, a], \quad \mu_{0} \leq \varphi .
$$

A upper solution for the problem (1) is a function $\mu \in C\left([0, a], E^{d}\right) \cap C^{1}\left([-\sigma, a], E^{d}\right)$ such that

$$
\mu^{\prime}(t) \geq f\left(t, u_{t}\right), \quad t \in[0, a], \quad \mu_{0} \geq \varphi
$$


Remark 3.1 If $\mu$ is (i)-differentiable (or (ii)-differentiable), then $\mu$ is said a lower (i)-solution (or a lower (ii)-solution), respectively. If $\mu$ is (i)-differentiable (or (ii)-differentiable), then $\mu$ is said an upper (i)-solution (or (ii)-differentiable), respectively.

Theorem 3.2 Suppose that there exists $\mu \in C\left([0, a], E^{d}\right) \cap C^{1}\left([-\sigma, a], E^{d}\right)$ a lower (i)solution of the problem (1). Let $f:[0, a] \times C_{\sigma} \rightarrow E^{d}$ be continuous such that

(i) $f$ is non-decreasing in the second variable, i.e., if $\varphi \geq \phi$ then $f(t, \varphi) \geq f(t, \phi)$;

(ii) $f$ is weakly contractive for comparable elements, i.e., for some altering distance functions $\Lambda$ and $\Upsilon$, it holds

$$
\Lambda\left(d_{\infty}(f(t, \varphi), f(t, \phi))\right) \leq \Lambda\left(D_{\sigma}(\varphi, \phi)\right)-\Upsilon\left(D_{\sigma}(\varphi, \phi)\right), \text { if } \varphi \geq \phi,
$$

for every $t \in[0, a]$ and $\varphi, \phi \in C_{\sigma}$.

Then, the problem (1) has a unique (i)-solution on $[-\sigma, a]$.

Proof. We consider the space $C\left([-\sigma, a], E^{d}\right)$ equipped with the complete metric

$$
D_{\rho}(u, v)=\sup _{t \in[-\sigma, a]}\left\{d_{\infty}(u(t), v(t)) e^{-\rho t}\right\}, \quad u, v \in C\left([-\sigma, a], E^{d}\right), \quad \text { for } \rho>0 .
$$

It is easy to see that this metric is equivalent to the metric $D(u, v)$, because

$$
D(u, v) e^{-\rho a} \leq D_{\rho}(u, v) \leq D(u, v)
$$

for all $u, v \in C\left([-\sigma, a], E^{d}\right)$. Moreover, $\left(C\left([-\sigma, a], E^{d}\right), D_{\sigma}\right)$ is a complete metric space.

Now, let the operator $\mathscr{T}: C\left([-\sigma, a], E^{d}\right) \rightarrow C\left([-\sigma, a], E^{d}\right)$ be defined by

$$
[\mathscr{T} u](t)= \begin{cases}\varphi(t), & \text { for } t \in[-\sigma, 0] \\ \varphi(0)+\int_{0}^{t} f\left(s, u_{s}\right) d s, & \text { for } t \in[0, a]\end{cases}
$$

If there exists $u \in C\left([-\sigma, a], E^{d}\right)$ is a fixed point of $\mathscr{T}$, then $u \in C^{1}\left([0, a], E^{d}\right)$ is a solution of the problem (1) and conversely.

In order to prove the theorem, we shall show the conditions of Theorem (2.1) are satisfied. First, we prove that the operator $\mathscr{T}$ is a non-decreasing and continuous. Indeed, the continuity is trivial and take $u \geq v$ on intervals $[0, a]$ and for every $t \in[-\sigma, 0]$, we have

$$
[\mathscr{T} u](t)=[\mathscr{T} v](t)=\varphi(t) .
$$

For every $t \in[0, a]$, we obtain

$$
[\mathscr{T} u](t)=\varphi(0)+\int_{0}^{t} f\left(s, u_{s}\right) d s \geq \varphi(0)+\int_{0}^{t} f\left(s, v_{s}\right) d s=[\mathscr{T} v](t) .
$$

From the assumption (ii), we have

$$
\Lambda\left(d_{\infty}(f(t, u), f(t, v))\right) \leq \Lambda\left(D_{\sigma}(u, v)\right), \quad \text { if } u, v \in C_{\sigma} \text { and } u \geq v .
$$

Next, we will prove that the inequality (6) holds, for all $u \geq v$. Suppose that

$$
D_{\sigma}(u, v)<d_{\infty}(f(t, u), f(t, v)), \quad \text { for all } u \geq v
$$

and $\Lambda$ is an altering distance function. Then we have

$$
\Lambda\left(D_{\sigma}(u, v)\right) \leq \Lambda\left(d_{\infty}(f(t, u), f(t, v))\right) .
$$


By the inequality (6) and (7), we derive that

$$
\Lambda\left(D_{\sigma}(u, v)\right)=\Lambda\left(d_{\infty}(f(t, u), f(t, v))\right), \quad \text { for all } u \geq v
$$

Combine assumption (ii) and (8), it follows that $0 \leq-\Upsilon\left(D_{\sigma}(u, v)\right)$, for all $u \geq$ $v$, and therefore, $\Upsilon\left(D_{\sigma}(u, v)\right)=0$. This implies $D_{\sigma}(u, v)=0$. Thus, we have $\Lambda\left(d_{\infty}(f(t, u), f(t, v))\right)=\Upsilon\left(D_{\sigma}(u, v)\right)=0$. Since $\Upsilon$ is an altering distance function, we derive that $d_{\infty}(f(t, u), f(t, v))=0$. This contradict the assumption above, that is, for all $u \geq v$ we have

$$
d_{\infty}(f(t, u), f(t, v)) \leq D_{\sigma}(u, v) .
$$

For all $u \geq v$, if $t \in[-\sigma, 0]$, then $d_{\infty}([\mathscr{T} u](t),[\mathscr{T} v](t))=d_{\infty}(\varphi(t), \varphi(t))=0$, and if $t \in[0, a]$, we get

$$
\begin{aligned}
d_{\infty}([\mathscr{T} u](t),[\mathscr{T} v](t)) & =d_{\infty}\left(\varphi(0)+\int_{0}^{t} f\left(s, u_{s}\right) d s, \varphi(0)+\int_{0}^{t} f\left(s, v_{s}\right) d s\right) \\
& \leq \int_{0}^{t} d_{\infty}\left(f\left(s, u_{s}\right), f\left(s, v_{s}\right)\right) d s \leq \int_{0}^{t} D_{\sigma}(u, v) d s \\
& \leq \int_{0}^{t} \sup _{r \in[-\sigma, 0]} d_{\infty}(u(r+s), v(r+s)) d s
\end{aligned}
$$

then

$$
\begin{aligned}
D_{\rho}(\mathscr{T} u, \mathscr{T} v) & =\sup _{t \in[-\sigma, a]}\left\{d_{\infty}([\mathscr{T} u](t),[\mathscr{T} v](t)) e^{-\rho t}\right\} \\
& \leq \sup _{t \in[-\sigma, a]}\left\{\int_{0}^{t} \sup _{r \in[-\sigma, 0]} d_{\infty}(u(r+s), v(r+s)) d s e^{-\rho t}\right\} \\
& =\sup _{t \in[-\sigma, a]}\left\{\int_{0}^{t} \sup _{r \in[-\sigma, 0]} d_{\infty}(u(r+s), v(r+s)) e^{-\rho(r+s)} e^{\rho(r+s)} d s e^{-\rho t}\right\} \\
& \leq \sup _{t \in[-\sigma, a]}\left\{\int_{0}^{t} \sup _{r \in[-\sigma, 0]} e^{\rho(r+s)} d s e^{-\rho t}\right\} D_{\rho}(u, v) \\
& =\sup _{t \in[-\sigma, a]}\left\{\int_{0}^{t} e^{\rho s} d s e^{-\rho t}\right\} D_{\rho}(u, v) \\
& =\sup _{t \in[-\sigma, a]}\left\{\frac{1-e^{-\rho t}}{\rho}\right\} D_{\rho}(u, v)=\frac{1-e^{-\rho a}}{\rho} D_{\rho}(u, v)
\end{aligned}
$$

Since $\Lambda$ is an altering distance function and by the inequality (10), we infer that

$$
\begin{aligned}
\Lambda\left(D_{\rho}(\mathscr{T} u, \mathscr{T} v)\right) & \leq \Lambda\left(\frac{1-e^{-\rho a}}{\rho} D_{\rho}(u, v)\right) \\
& =\Lambda\left(D_{\rho}(u, v)\right)-\left(\Lambda\left(D_{\rho}(u, v)\right)-\Lambda\left(\frac{1-e^{-\rho a}}{\rho} D_{\rho}(u, v)\right)\right)
\end{aligned}
$$

Therefore, if $\Upsilon(t)=\Lambda(t)-\Lambda\left(\frac{1-e^{-\rho a}}{\rho} t\right)$, it follows that

$$
\Lambda\left(D_{\rho}(\mathscr{T} u, \mathscr{T} v)\right) \leq \Lambda\left(D_{\rho}(\varphi, \phi)\right)-\Upsilon\left(D_{\rho}(\varphi, \phi)\right), \quad \text { for all } u \geq v
$$


From 4 and (8), we obtain

$$
\Lambda\left(d_{\infty}(f(t, u), f(t, v))\right) \leq \Lambda\left(D_{\sigma}(\varphi, \phi)\right)-\Upsilon\left(D_{\sigma}(\varphi, \phi)\right), \quad \text { for all } u \geq v .
$$

Finally, using the existence of the lower (i)-solution and Theorem 2.1, we shall prove that $\mu$ is such that $\mu \leq \mathscr{T} \mu$. Indeed, if $t \in[-\sigma, 0], \mu_{0} \leq \varphi$ then $\mu(t) \leq \varphi(t)=[\mathscr{T} \mu](t)$ and for $t \in[0, a]$,

$$
\mu(t)=\mu(0)+\int_{0}^{t} \mu^{\prime}(s) d s \leq \varphi(0)+\int_{0}^{t} f\left(s, \mu_{s}\right) d s=[\mathscr{T} \mu](t) .
$$

Since every pair of functions in $C\left([-\sigma, a], E^{d}\right)$ has an upper bound, the operator $\mathscr{T}$ has a unique fixed point $u \in C\left([-\sigma, a], E^{d}\right)$ and $u$ is the unique solution of the problem (1) on $[-\sigma, a]$.

Theorem 3.3 Replacing the existence of a lower (i)-solution of the problem (1) by the existence of a upper (i)-solution of the problem (1), the conclusion of Theorem 3.2 is still valid.

Proof. The proof of Theorem 3.3 is similarly the proof Theorem 3.2.

Theorem 3.4 Suppose that there exists $\mu \in C\left([0, a], E^{d}\right) \cap C^{1}\left([-\sigma, a], E^{d}\right)$ a lower (ii)solution of the problem (1). Let $f:[0, a] \times C_{\sigma} \rightarrow E^{d}$ be continuous such that

(i) for all $\alpha \in[0,1]$ and $\varphi \in C_{\sigma}$,

$$
\operatorname{diam}\left([\varphi(0)]^{\alpha}\right) \geq \operatorname{diam}\left(\left[\int_{t_{0}}^{t} f\left(s, u_{s}\right) d s\right]^{\alpha}\right)
$$

(ii) $f$ is non-decreasing in the second variable, i.e., if $\varphi \geq \phi$ then $f(t, \varphi) \geq f(t, \phi)$;

(iii) $f$ is weakly contractive for comparable elements, i.e., for some altering distance functions $\Lambda$ and $\Upsilon$, it holds

$$
\Lambda\left(d_{\infty}(f(t, \varphi), f(t, \phi))\right) \leq \Lambda\left(D_{\sigma}(\varphi, \phi)\right)-\Upsilon\left(D_{\sigma}(\varphi, \phi)\right), \text { if } \varphi \geq \phi,
$$

for every $t \in[0, a]$ and $\varphi, \phi \in C_{\sigma}$.

Then, the problem (1) has a unique (ii)-solution on $[-\sigma, a]$.

Proof. By the condition (i), we imply that the existence of Hukuhara differences $\varphi(0)+(-1) \int_{0}^{t} f\left(s, u_{s}\right) d s$ is guaranteed. Now, let the operator $\mathscr{A}: C\left([-\sigma, a], E^{d}\right) \rightarrow$ $C\left([-\sigma, a], E^{d}\right)$ be defined by

$$
[\mathscr{A} u](t)= \begin{cases}\varphi(t), & \text { for } t \in[-\sigma, 0] \\ \varphi(0) \ominus(-1) \int_{0}^{t} f\left(s, u_{s}\right) d s, & \text { for } t \in[0, a]\end{cases}
$$

Notice that if there exists $u \in C\left([-\sigma, a], E^{d}\right)$ is a fixed point of $\mathscr{A}$, then $u \in C^{1}\left([0, a], E^{d}\right)$ is a solution of the problem (1) and conversely.

Similarly to Theorem 3.2, we shall show the conditions of Theorem (2.1) are satisfied. For all $u_{s} \geq v_{s}$ on $[-\sigma, a]$, we have

$$
[\mathscr{A} u](t)=[\mathscr{A} v](t)=\varphi(t) \text { for } t \in[-\sigma, 0]
$$


and

$[\mathscr{A} u](t)=\varphi(0) \ominus(-1) \int_{0}^{t} f\left(s, u_{s}\right) d s \geq \varphi(0) \ominus(-1) \int_{0}^{t} f\left(s, v_{s}\right) d s=[\mathscr{A} v](t), \quad$ for $t \in[0, a]$.

Therefore, $\mathscr{A}$ has the operator non-decreasing.

For all $u \geq v$, if $t \in[-\sigma, 0]$ then $d_{\infty}([\mathscr{A} u](t),[\mathscr{A} v](t))=d_{\infty}(\varphi(t), \varphi(t))=0$ and if $t \in[0, a]$, then we get

$$
\begin{aligned}
d_{\infty}([\mathscr{A} u](t),[\mathscr{A} v](t)) & =d_{\infty}\left(\varphi(0) \ominus(-1) \int_{0}^{t} f\left(s, u_{s}\right) d s, \varphi(0) \ominus(-1) \int_{0}^{t} f\left(s, v_{s}\right) d s\right) \\
& \leq \int_{0}^{t} d_{\infty}\left(f\left(s, u_{s}\right), f\left(s, v_{s}\right)\right) d s \leq \int_{0}^{t} D_{\sigma}\left(u_{s}, v_{s}\right) d s \\
& \leq \int_{0}^{t} \sup _{r \in[-\sigma, 0]} d_{\infty}(u(r+s), v(r+s)) d s .
\end{aligned}
$$

By similar calculations as (10), we have.

$$
\begin{aligned}
D_{\rho}(\mathscr{T} u, \mathscr{T} v) & =\sup _{t \in[-\sigma, a]}\left\{d_{\infty}([\mathscr{T} u](t),[\mathscr{T} v](t)) e^{-\rho t}\right\} \\
& \leq \sup _{t \in[-\sigma, a]}\left\{\frac{1-e^{-\rho t}}{\rho}\right\} D_{\rho}(u, v)=\frac{1-e^{-\rho a}}{\rho} D_{\rho}(u, v) .
\end{aligned}
$$

Therefore, if $\Lambda$ is some increasing altering distance function, it holds

$$
\begin{aligned}
\Lambda\left(D_{\rho}(\mathscr{T} u, \mathscr{T} v)\right) & \leq \Lambda\left(\frac{1-e^{-\rho a}}{\rho} D_{\rho}(u, v)\right) \\
& =\Lambda\left(D_{\rho}(u, v)\right)-\left(\Lambda\left(D_{\rho}(u, v)\right)-\Lambda\left(\frac{1-e^{-\rho a}}{\rho} D_{\rho}(u, v)\right)\right)
\end{aligned}
$$

Then, from 4 and (8), we derive that

$$
\Lambda\left(d_{\infty}(f(t, u), f(t, v))\right) \leq \Lambda\left(D_{\sigma}(\varphi, \phi)\right)-\Upsilon\left(D_{\sigma}(\varphi, \phi)\right), \quad \text { for all } u \geq v .
$$

where $\Upsilon(t)=\Lambda(t)-\Lambda\left(\frac{1-e^{-\rho a}}{\rho} t\right)$.

Finally, using the existence of the lower (ii)-solution and Theorem 3.1, we shall prove that $\mu$ is such that $\mu \leq \mathscr{A} \mu$. Indeed, if $t \in[-\sigma, 0], \mu_{0} \leq \varphi$ then $\mu(t) \leq \varphi(t)=[\mathscr{A} \mu](t)$ and for $t \in[0, a]$,

$$
\mu(t)=\mu(0) \ominus(-1) \int_{0}^{t} \mu^{\prime}(s) d s \leq \varphi(0) \ominus(-1) \int_{0}^{t} f\left(s, \mu_{s}\right) d s=[\mathscr{A} \mu](t) .
$$

Thus $\mu(t) \leq[\mathscr{A} \mu](t)$. We see that the operator $\mathscr{A}$ verifies all conditions of Theorem 2.1, that is, $\mathscr{A}$ has a fixed point in $C\left(\left[-\sigma, E^{d}\right]\right)$. Given that $C\left(\left[-\sigma, E^{d}\right]\right)$ verifies that every pair of elements of $C\left(\left[-\sigma, E^{d}\right]\right)$ has an upper bound, the operator $\mathscr{A}$ has a unique fixed point.

Theorem 3.5 Replacing the existence of a lower (ii)-solution of the problem (11) by the existence of a upper (ii)-solution of the problem (11), the conclusion of Theorem 3.5 is still valid. 
Proof. The proof of Theorem 3.5 is similarly the proof Theorem 3.4.

Example 3.6 Let us consider a fuzzy time-delay Malthusian model:

$$
\begin{cases}u^{\prime}(t)=\lambda u(t-1), & t \geq 0 \\ u(t)=u_{0}, & -1 \leq t \leq 0\end{cases}
$$

where $u(t)$ is the population at time $t, u_{0}=(-1,0,1)$ and $\lambda>0$.

In $[12,13]$, it is shown that the problem (13) has a unique solution on $[-1, \infty)$.

But this example, we want to prove that the conditions of Theorem 3.2 (or Theorem 3.2) are satisfied.

Now, we check the existence of a lower (i)-solution for the problem (13). Let $f:[0, \infty) \times$ $C\left([-1,0], E^{1}\right) \rightarrow E^{1}$ be defined by $f(t, u)=\lambda u(t-1)$ is a continuous and non-increasing in the second variable, that is,

$$
f(t, u)=\lambda u(t-1) \geq f(t, v)=\lambda v(t-1), \quad \text { if } u \geq v, \quad \text { for } t \in[0, \infty) .
$$

Let the function $\Lambda, \Upsilon: \mathbb{R}_{+} \rightarrow \mathbb{R}_{+}$be defined by $\Lambda(t)=t$ and $\Upsilon(t)=(1-\lambda) t$. It is easy to see that $\Lambda, \Upsilon$ satisfy the conditions of Definition 2.1, that is, $\Lambda$ and $\Upsilon$ are some altering distance function.

By a direct calculation, we get

$$
\begin{aligned}
d_{\infty}(f(t, u), f(t, v)) & =\lambda d_{\infty}(u(t-1), v(t-1)) \\
& =\lambda \sup _{\alpha \in[0,1]} \max \left\{\left|u_{\alpha}^{l}(t-1)-v_{\alpha}^{l}(t-1)\right|,\left|u_{\alpha}^{r}(t-1)-v_{\alpha}^{r}(t-1)\right|\right\} \\
& =\lambda \sup _{\alpha \in[0,1]} \max \left\{\sup _{s \in[-1,0]}\left|u_{\alpha}^{l}(s)-v_{\alpha}^{l}(s)\right|, \sup _{s \in[-1,0]}\left|u_{\alpha}^{r}(s)-v_{\alpha}^{r}(s)\right|\right\} \\
& =\lambda \sup _{\alpha \in[0,1]}\left\{\sup _{s \in[-1,0]} d_{H}\left([u(s)]^{\alpha},[v(s)]^{\alpha}\right)\right\} \\
& \leq \lambda \sup _{s \in[-1,0]} d_{\infty}(u(s), v(s))=\lambda D_{\sigma}(u, v) .
\end{aligned}
$$

Since $\Lambda$ is an altering distance function and from (15), we infer that

$$
\begin{aligned}
\Lambda\left(d_{\infty}(f(t, u), f(t, v))\right) & \leq \Lambda\left(\lambda D_{\sigma}(u, v)\right) \\
& \leq \Lambda\left(D_{\sigma}(u, v)\right)-\left(\Lambda\left(D_{\sigma}(u, v)\right)-\Lambda\left(\lambda D_{\sigma}(u, v)\right)\right) \\
& \leq \Lambda\left(D_{\sigma}(u, v)\right)-\Upsilon\left(D_{\sigma}(u, v)\right),
\end{aligned}
$$

where $\Upsilon(t)=(1-\lambda) t$.

By using Theorem 3.2, the existence of a lower (i)-solution for the problem provides the existence of a unique solution on $[-1, \infty)$.

It is easy to see that the conditions of Theorem (3.3) are satisfied, that is, the problem (13) has a unique (ii)-solution on $[-1, \infty)$.

Example 3.7 Consider the fuzzy functional differential equation that describe the fuzzy Ehrlich ascities tumor model

$$
\begin{cases}u^{\prime}(t)=r u(t-1)(1-u(t-1)), & t \geq 0, \\ u(t)=u_{0}, & t \in[-1,0]\end{cases}
$$

where $u_{0}=(-1,0,1)$ and $r \in[0,2)$. 
Similar to example above, we can easily see that all the conditions of Theorem (3.2) are verified and we can conclude the existence and uniqueness of solution of problem (17) by means of Theorem (3.2), though the unique solution for problem (17).

\section{Conclusions}

In this paper, we studied the new existence and uniqueness of solution for fuzzy functional differential equation under generalized Hukuhara derivative and by using some recent results of fixed point of weakly contractive mappings on partially ordered sets, established in [18]. We obtain a generalization of these results, if we replace the condition (h1) of Theorem 4.1 in [13] by the following condition $\Lambda\left(d_{\infty}(f(t, \varphi), f(t, \phi))\right) \leq \Lambda\left(D_{\sigma}(\varphi, \phi)\right)-\Upsilon\left(D_{\sigma}(\varphi, \phi)\right)$, where $\Lambda, \Upsilon$ are some altering distance functions.

\section{References}

[1] V. Lakshmikantham, R.N. Mohapatra, Theory of Fuzzy Differential Equations and Inclusions (Taylor and Francis Publishers, London, 2003), ISBN 9780415300735

[2] S.S.L. Chang, L.A. Zadeh (World Scientific Publishing Co., Inc., River Edge, NJ, USA, 1996), chap. On Fuzzy Mapping and Control, pp. 180-184, ISBN 981-02-2422-2, http://dl . acm.org/citation. cfm?id=234347.234388

[3] D. Dubois, H. Prade, Fuzzy Sets and Systems 8, 1 (1982)

[4] D. Dubois, H. Prade, Fuzzy Sets and Systems 8, 105 (1982)

[5] L. Zadeh, Information and Control 8, 338 (1965)

[6] M.L. Puri, D.A. Ralescu, Journal of Mathematical Analysis and Applications 91, 552 (1983)

[7] O. Kaleva, Fuzzy Sets and Systems 24, 301 (1987), fuzzy Numbers

[8] S. Song, C. Wu, Fuzzy Sets and Systems 110, 55 (2000)

[9] C. Wu, S. Song, E. Lee, Journal of Mathematical Analysis and Applications 202, 629 (1996)

[10] B. Bede, S.G. Gal, Fuzzy Sets Syst. 151, 581 (2005)

[11] S.S. Behzadi, T. Allahviranloo, Iranian Journal of Fuzzy Systems 13, 71 (2016)

[12] A. Khastan, J. Nieto, R. Rodríguez-López, Information Sciences 275, 145 (2014)

[13] V. Lupulescu, Fuzzy Sets and Systems 160, 1547 (2009)

[14] E.J. Villamizar-Roa, V. Angulo-Castillo, Y. Chalco-Cano, Fuzzy Sets and Systems 265, 24 (2015)

[15] O.S. Fard, D.F.M. Torres, M.R. Zadeh, Applicable Analysis and Discrete Mathematics pp. 152-167 (2016)

[16] P. Subrahmanyam, S. Sudarsanam, Fuzzy Sets and Systems 81, 237 (1996)

[17] T. Allahviranloo, P. Salehi, M. Nejatiyan, Iranian Journal of Fuzzy Systems 12, 75 (2015)

[18] J. Harjani, K. Sadarangani, Nonlinear Analysis: Theory, Methods \& Applications 72, 1188 (2010) 